\title{
BMJ Open Study protocol for a single-blind, placebo-controlled randomised trial of Tianjiu effects in patients with intradialytic hypotension
}

\author{
Ming-Yen Tsai, ${ }^{1,2}$ Yu-Jen Su, ${ }^{3}$ Hwee-Yeong Ng, ${ }^{3}$ Shih-Yu Chen, ${ }^{4}$ \\ Yu-Chuen Huang, ${ }^{5}$ Chien-Hsing Wu, ${ }^{3}$ Yung-Hsiang Chen ${ }^{1,6}$
}

To cite: Tsai M-Y, Su Y-J, $\mathrm{Ng} \mathrm{H}-\mathrm{Y}$, et al. Study protocol for a single-blind, placebocontrolled randomised trial of Tianjiu effects in patients with intradialytic hypotension. BMJ Open 2016;6:e009976. doi:10.1136/bmjopen-2015009976

- Prepublication history for this paper is available online. To view these files please visit the journal online (http://dx.doi.org/10.1136/ bmjopen-2015-009976)

C-HW and Y-HC contributed equally to this study.

Received 11 September 2015 Revised 15 February 2016 Accepted 19 February 2016

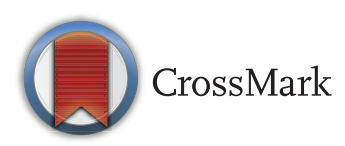

For numbered affiliations see end of article.

\section{Correspondence to} Chien-Hsing Wu; chienhsingwu@gmail.com and Yung-Hsiang Chen; yhchen@mail.cmu.edu.tw

\section{ABSTRACT}

Introduction: Intradialytic hypotension (IDH) is the most frequent complication of haemodialysis (HD) and may contribute to cardiovascular events and high mortality. The aetiology of IDH is multifactorial; therefore, it remains a challenging problem in the management of patients with $\mathrm{HD}$. Since the application of Tianjiu at specific points can influence haemodynamics, we hypothesise that Tianjiu therapy at the traditionally used meridian points will reduce the severity of hypotension in patients who undergo HD.

Methods/analysis: In this clinical trial, eligible patients with IDH will be divided randomly and equally into a Tianjiu group and a control group for 4 weeks. In the Tianjiu group, the patients will have Tianjiu applied at three points (conception vessel 4 , and bilateral kidney 1) during each HD session. In the control group, patients will have clay patches applied in the same way as those in the Tianjiu treatment group. Both groups will be followed up for 2 weeks. The primary outcome measure will be the percentage of target ultrafiltration achieved, defined as the actual ultrafiltration volume divided by the target ultrafiltration volume. Secondary outcome measures, including frequency of IDH episodes and number of nursing interventions during $H D$ sessions, predialysis and postdialysis blood pressure (BP), patient's participative assessment of the degree of fatigue after dialysis (scale from 0 , not at all, to 10 , extremely), and recovery time from fatigue after dialysis will be recorded at the 0th and 4th weeks.

Ethics/dissemination: This trial has undergone ethical scrutiny and been approved by the ethics review board of Chang Gung Memorial Hospital (Permission number: $102-4749 \mathrm{~A} 3$ and 104-3156C). The pre-results of this trial will help to determine whether Tianjiu is an effective and safe treatment for IDH, and, if so, whether it is a therapeutic effect rather than a placebo effect.

Trial registration number: NCT02210377; Pre-results.

\section{INTRODUCTION}

Intradialytic hypotension (IDH) has been reported in $20-30 \%$ of patients treated with maintenance haemodialysis $\quad(\mathrm{HD}){ }^{1} \quad$ The symptoms associated with this clinical problem, such as nausea, dizziness and cramps, can have a negative impact on patients' quality of life and tolerability to dialysis. ${ }^{2}$ In addition, IDH can increase patients' morbidity and mortality by aggravating the risk of cardiovascular complications, mesenteric or cerebral ischaemia, suboptimal dialysis adequacy and ultrafiltration (UF), and left ventricular hypertrophy. ${ }^{3-5}$

The aetiology of IDH is multifactorial. The main factor is rapid removal of intravascular volume by UF and the subsequent imbalance between UF and the plasma refilling rate. ${ }^{6}$ Another possible contributor to IDH is impaired cardiovascular compensation for the reduced circulating volume, which includes increased cardiac output and contractility and increased peripheral vascular resistance. ${ }^{78}$ Other factors, such as the rapid reduction in plasma osmolality, autonomic dysfunction and increased synthesis of endogenous vasodilators, have also been reported to be associated with haemodynamic instability. ${ }^{9-11}$

Several approaches have been applied to prevent and manage IDH. These include accurate assessment of dry weight, avoidance of excessive interdialytic weight gain, fasting during dialysis, adequate adjustment of antihypertension agents, sodium and UF profiling, cooling of the dialysate, use of the dialysate with a bicarbonate buffer or a high calcium content, pharmacological measures including $\alpha 1$-adrenergic agonist, and convective therapies, including haemofiltration and haemodiafiltration. ${ }^{12-15}$

Tianjiu (also called crude herb moxibustion, automoxibustion, herbal acupoint paste and cold moxibustion therapy) is one type of moxibustion that is widely used in Asian 
countries as traditional Chinese medicine (TCM). It is a permeability treatment that involves pasting Chinese herbs as an irritant near acupoints to cause a warm and painless sensation. ${ }^{16}$ The general theory of Tianjiu is based on the generation of the warm meridian energy (Yang-Qi), which is believed to be responsible for chronic diseases associated with cold-deficiency syndrome and poor immunity. ${ }^{17}$ Tianjiu therapy has been used to modulate autonomic nervous activity, neurotransmitter levels, endogenous substance levels and levels of inflammatory factors, and to strengthen cerebral, cardiovascular and renal function. ${ }^{18-21}$

In TCM theory, symptomatic IDH is aetiologically caused by the derangement of Qi-blood and subsequent prostration of Yang-Qi in the human body during the rapid fluid removal of HD. Accumulating evidence shows that applying moxibustion to specific acupoints has a therapeutic effect on clinical symptoms and quality of life in patients with HD. ${ }^{22} 23$ Tianjiu, one moxibustion therapy, also features a stimulating effect. Therefore, it should make the Yang-Qi abundant, blood circulation strong, and autonomic nerve activity smooth and harmonised in patients with HD. Unfortunately, clinical trials examining Tianjiu therapy for IDH are lacking.

This proposal describes a protocol for a randomised controlled trial (RCT) that aims to test the efficacy and safety of Tianjiu therapy during HD sessions, and to determine whether the intervention can reduce the frequency of symptomatic IDH episodes and IDH-related interventions and improve dialysis adequacy, volume control, blood pressure and quality of life. The results of this study will provide evidence for assessing the need for a large clinical trial and yield data to determine the appropriate sample size for future large-scale RCTs of Tianjiu therapy in patients with IDH.

\section{METHODS AND ANALYSIS}

\section{Study design}

A randomised, single-blinded controlled trial of Tianjiu for the treatment of IDH in patients undergoing dialysis is currently being conducted at Kaohsiung Chang Gung Memorial Hospital (KCGMH), Taiwan. All screening appointments and study visits will occur in the HD units for outpatients. This study has been ethically approved by the Institutional Review Board of CGMH and is registered with the Ethics approval numbers 102-4749A3 and 104-3156C.

The total length of the trial at KCGMH will be 2 years, from March 2014 to July 2016. We plan to recruit participants via advertisements in our HD units. Eligible participants will be identified by a nephrologist as meeting the criteria of IDH in this study and will be randomly and equally assigned to the Tianjiu group or the placebo group at a 1:1 ratio. Randomisation will be generated by a computerised random number function in Microsoft Excel, and the patients, programme assessors and statisticians will be unaware of the group to which they have been assigned. A block randomisation procedure (based on age, comorbidities such as cardiovascular disease and diabetes mellitus) will be employed to ensure that group allocation is equal and that the characteristics of the trial participants are similar. Only the research assistant will know to which group each patient belongs for regulation of the application of the methods. The duration of the study will be 7 weeks, including a baseline period of 1 week (week $0-1$ ), a treatment period of 4 weeks (week 1-4), and a follow-up period of 2 weeks (week 5-6). A flow chart of the trial procedure is presented in figure 1 .

\section{Setting}

This clinical trial will be conducted in a single centre, with the participant blinded to treatment allocation. All patients diagnosed with IDH in accordance with the National Kidney Foundation's Kidney Disease Outcomes Quality Initiative (KDOQI) guidelines will be enrolled in this trial. ${ }^{3}$ Symptomatic hypotension is defined as a drop in SBP of $>20 \mathrm{~mm} \mathrm{Hg}$ from baseline or absolute SBP $<100 \mathrm{~mm} \mathrm{Hg}$ accompanied by at least one of the following: diaphoresis, nausea, vomiting, cramps, headache or dizziness. The whole treatment process will be carried out at Kaohsiung Chang Gung Memorial Hospital (KCGMH), Taiwan.

\section{Participants}

All participants who have been treated with HD for more than 3 months will be screened for eligibility. To ensure blinding, only patients who have never received Tianjiu before participating in this study will be included. Other inclusion criteria for the study are as follows: age between 20 and 75 years, medically stable condition, thrice-weekly treatment with maintenance HD for $4 \mathrm{~h}$ per session, and $>15 \%$ of HD sessions in the preceding 2 months complicated by symptomatic IDH.

Participants with concurrent severe disorders of the heart, brain, liver or the haematopoietic system; active malignancy; mental disorders; pregnancy or lactation; hypersensitivity skin reactions following Tianjiu therapy; or any other condition that the investigator judges as likely to make the patient unable to complete or comply with, or otherwise unsuitable for, the study will be excluded. Informed consent will be obtained from each individual who agrees to participate in the study. In addition, patients who are hospitalised for other reasons or who experience hypertensive crisis (defined as $\mathrm{SBP}>180 \mathrm{~mm} \mathrm{Hg}$ or DBP $>120 \mathrm{~mm} \mathrm{Hg}$ ) ${ }^{24}$ or other adverse events during the treatment period will be excluded from Tianjiu intervention.

\section{Interventions}

Following enrolment, participants will undergo a 1-week baseline period for comprehensive clinical assessment including dry weight reduction, medication review and standardisation of their dialysis prescription. After randomisation, patients in both groups will receive 12 
Figure 1 Flow chart of study procedure.

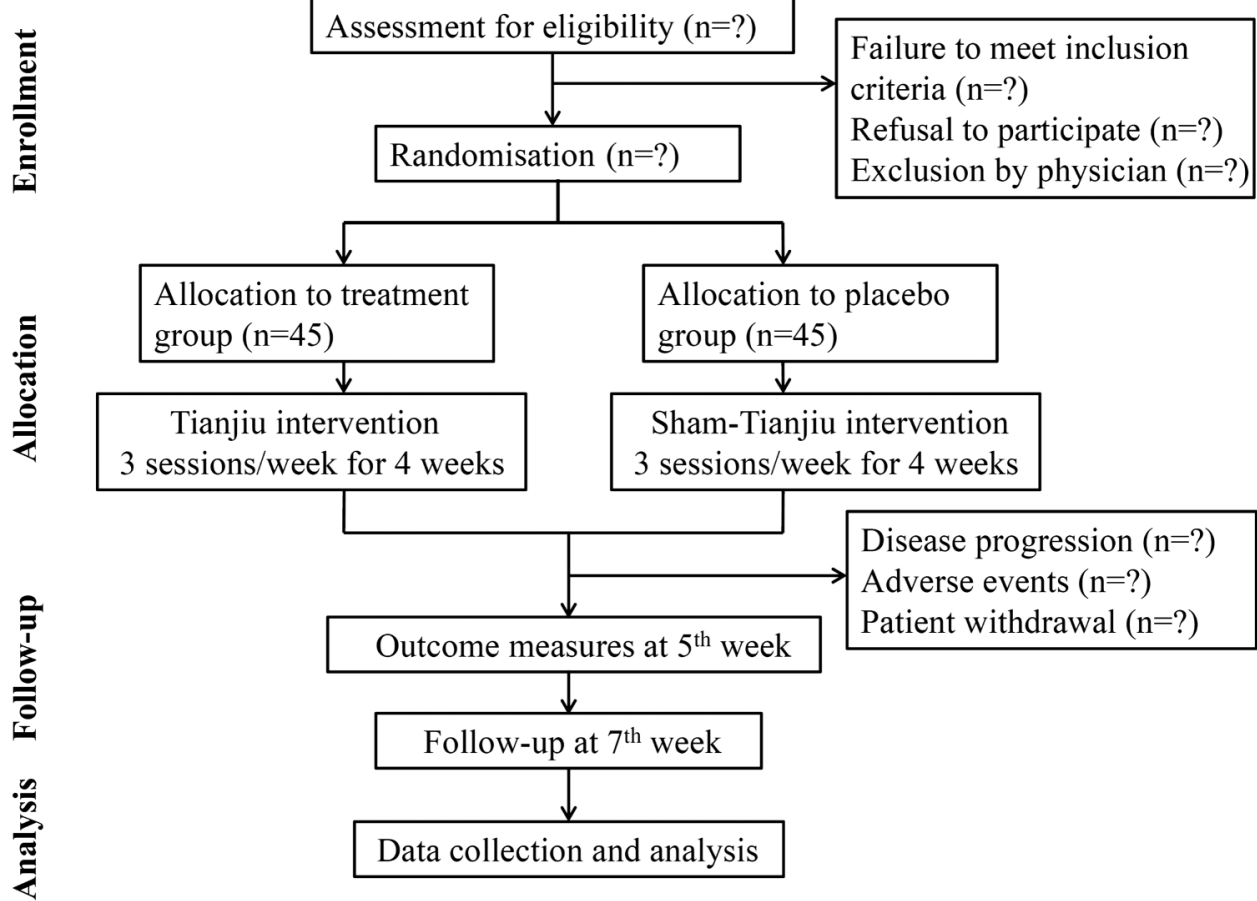

sessions of treatment over a 4-week treatment period. The dialysis prescription, dialysate, and artificial kidney machines will not be altered during the treatment period. UF volumes will be adapted to reach $<5 \%$ of dry weight during each HD session. Dry weight will be determined clinically by the patient's attending nephrologist. $\mathrm{Kt} / \mathrm{V}$ will be calculated using the Daugirdas secondgeneration logarithmic equation. ${ }^{25}$ The HD nurse assigned to each individual participant will record haemodynamic parameters, treatment parameters and IDH-related interventions on standard clinical HD run-sheets.

\section{Treatment group and placebo group}

The participants in the Tianjiu group will be treated with Chinese herbal patches at acupoints on the abdomen and plantar, three times per week, for $4 \mathrm{~h}$ each time during HD. A Tianjiu patch (diameter $2.0 \mathrm{~cm}$, depth $0.5 \mathrm{~cm}$ ) placed on each acupoint will be covered by gauze with a non-woven adhesive plaster in the middle (figure 2A), and the participants in the control group will be given placebo patches (brown clay patches) (figure 2B) on the same sites. The format of the placebo (clay) intervention will be the same as in the treatment group. Participants will be instructed to lie supine before their HD session, and then the bilateral KI1 (Yongquan) (figure 2C) and CV4 (Guanyuan) (figure 2D) will be selected on the basis of evaluation of moxibustion literature and will be disinfected using $75 \%$ alcohol. To reduce bias, the trial participants will be blinded to the intervention. Our HD nurse will be encouraged to supervise participants and to prevent them from touching the patches during each HD session. At the end of the HD, the patches will be removed by research assistants and the surrounding skin will be checked.

\section{Prescription and preparation of the Tianjiu paste}

The prescription of Tianjiu therapy employed in this study will be referenced from Zhang Shi Yi Tong (Zhang Lu from the Qing Dynasty). The regimens will mainly include Sinapis Semen, Corydalis Rhizoma, Euphorbiae Kansui Radix, Asari Herba Cum Radice and Boreneolum Syntheticum, which will be ground into powder in an ultrafine grinder and then mixed in suitable proportions of 10:10:5:5:1. ${ }^{26}$ Fresh ginger juice will be added to these herbs in a ratio of 1:1 before use. All herbs were provided by Sheng Chang Pharmaceutical Co., Ltd. in June 2014 and can be refrigerated for more than 2 years. The paste will be produced by the Chinese Medicine Pharmacy of KCGMH on the day of use.

Bilateral KI1 and CV4 will be selected on the basis of an evaluation of moxibustion literature, experts' recommendations and the clinical experience of the researchers. KI1 is located on the points of the $1 / 3$ and $2 / 3$ intersection of the plantar. KI1 has the function of opening the sensory orifices, calming the spirit, recovering from unconsciousness, discharging heat, stimulating the blood pressure and restoring yang to prevent collapse. $^{27} 28 \mathrm{CV} 4$ is located on the midline of the abdomen, $3 \mathrm{~cm}$ below the centre of the umbilicus, and belongs to the crossing acupoint of the conception vessel and the 3 Yin meridians. It is an important point for reducing exhaustion, promoting blood circulation, invigorating the kidney $\mathrm{Qi}$ and strengthening immunity. ${ }^{29}$

\section{Selection and application of acupoints}



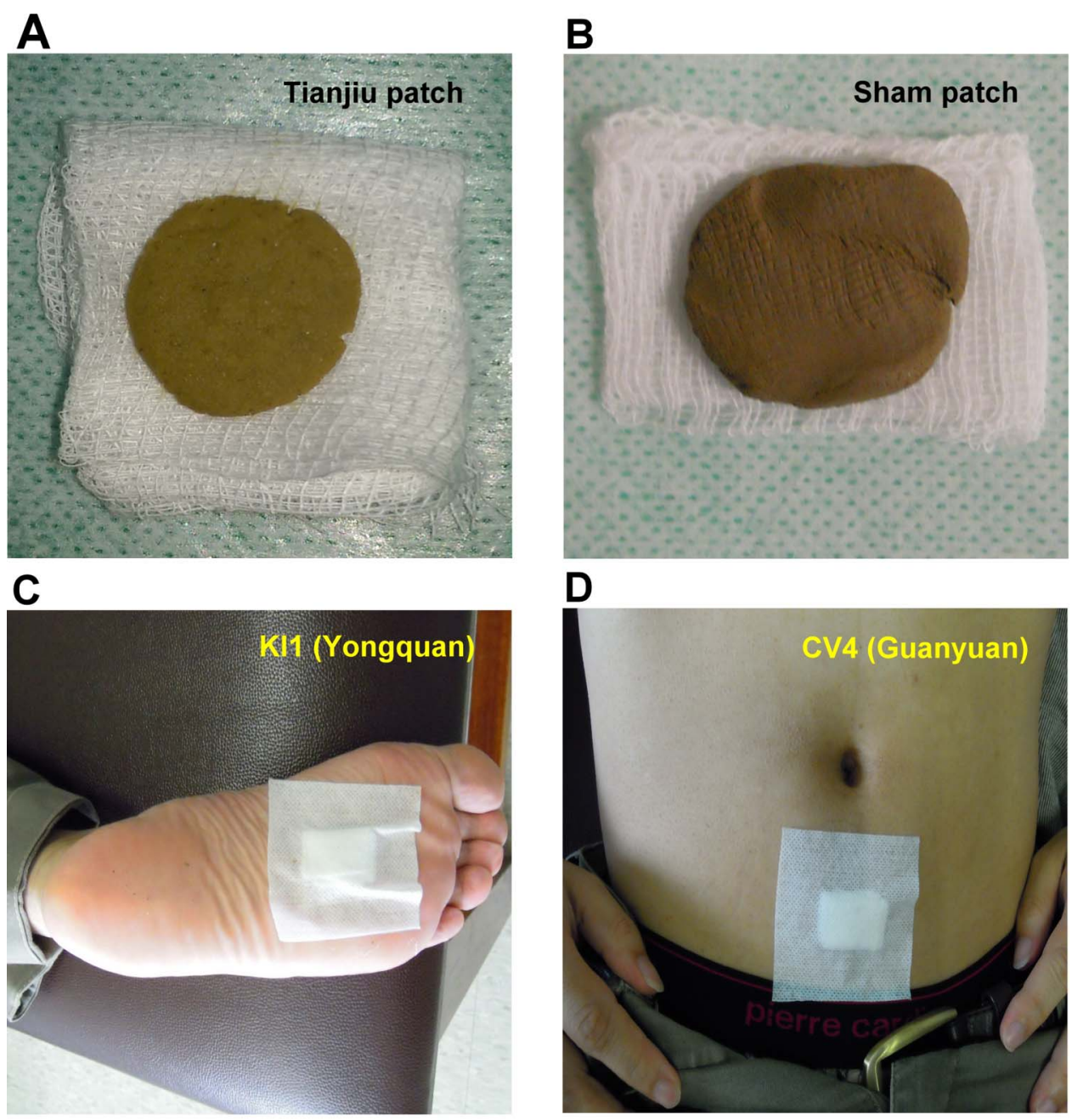

Figure 2 Photographs of the Tianjiu therapy that will be used in this trial. (A) Actual Tianjiu patch (a traditional Chinese method that uses the warmth and irritation generated by mixed herbal patches to stimulate acupoints); (B) sham patch (clay patches of the same colour and size); (C) therapeutic intervention of KI1 (Yongquan); (D) therapeutic intervention of CV4 (Guanyuan).

\section{Outcome measures}

The primary outcome measure will be the percentage of target UF achieved and will be defined as follows: \% Target UF achieved will be defined as actual UF volume/target UF volume. Target UF volume will be the difference between the predialysis weight and the dry weight. Actual UF volume will be the difference between the predialysis and postdialysis weights.

Secondary outcome measures (predialysis and postdialysis SBP, predialysis and postdialysis DBP, nadir SBP and nadir DBP, frequency of symptomatic IDH, and any IDH-related nursing interventions to treat hypotension episodes) will be recorded for each dialysis session. Blood pressure will be measured before dialysis, every 30 min during HD and after dialysis in each HD session of the study. IDH-related interventions will be defined as the use of the Trendelenburg position, manual reduction of UF rate, infusion of isotonic saline or hypertonic fluid, lowering of dialysate temperature, or dialysis cessation. Additionally, each patient's subjective assessment of the degree of fatigue after dialysis (scale from 0 , not at all, to 10, extreme) and recovery time from fatigue after dialysis (within minutes, when arriving home, at bed time, the next morning, by next HD) will be measured at the 0th and 4th weeks of the study period. Data on dry weight, haematocrit and serum albumin will be collected for all periods of the study. The outcome measurement time points are provided in detail in table 1.

\section{Quality control and data collection}

All staff involved in the trial will receive training before implementation of the trial. The training programme will include case screening and recruitment, the intervention method, outcome measures and data processing. The research assistants will check study protocol compliance and informed consent documents and assess the progress of the study, including participant randomisation, Tianjiu patch intervention and data quality. Dropouts and withdrawals from the study will be recorded throughout the intervention and follow-up periods. 
Table 1 Timing of visits and data collection

\begin{tabular}{|c|c|c|c|c|c|}
\hline & \multirow{2}{*}{$\begin{array}{l}\text { Screening } \\
1 \text { week }\end{array}$} & \multirow{2}{*}{$\begin{array}{l}\text { Baseline } \\
\text { period } \\
\text { 0-week }\end{array}$} & \multicolumn{2}{|c|}{ Treatment period } & \multirow{2}{*}{$\begin{array}{l}\text { Follow-up } \\
\text { period } \\
\text { 6-week }\end{array}$} \\
\hline & & & 1 week & 4-week & \\
\hline \multicolumn{6}{|l|}{ Patient } \\
\hline Eligibility & $\mathrm{X}$ & & & & \\
\hline Informed consent & & $X$ & & & \\
\hline Demographics and medical history & & $\mathrm{X}$ & & & \\
\hline Physical examination & & $\mathrm{X}$ & & & \\
\hline Randomisation & & & $\mathrm{X}$ & & \\
\hline \multicolumn{6}{|l|}{ Intervention } \\
\hline Treatment group & & & $\begin{array}{l}12 \text { sess } \\
\text { at acupc }\end{array}$ & f Tianjiu & \\
\hline \multicolumn{6}{|l|}{ Comparison } \\
\hline Control group & & & $\begin{array}{l}12 \text { sess } \\
\text { Tianjiu }\end{array}$ & $\begin{array}{l}\text { f sham- } \\
\text { points }\end{array}$ & \\
\hline \multicolumn{6}{|l|}{ Outcomes } \\
\hline Blood pressure & & $X$ & $\mathrm{X}$ & $\mathrm{X}$ & $\mathrm{X}$ \\
\hline Per cent target ultrafiltration & & $\mathrm{X}$ & $\mathrm{X}$ & $X$ & $\mathrm{X}$ \\
\hline Total number of hypotension episodes & & $\mathrm{X}$ & $\mathrm{X}$ & $\mathrm{X}$ & $\mathrm{X}$ \\
\hline Total number of IDH-related nursing interventions & & $\mathrm{X}$ & $\mathrm{X}$ & $\mathrm{x}$ & $\mathrm{X}$ \\
\hline Laboratory test & & $\mathrm{X}$ & & & $\mathrm{X}$ \\
\hline Fatigue scale after dialysis & & $\mathrm{X}$ & & $\mathrm{X}$ & \\
\hline Recovery time from fatigue after dialysis & & $\mathrm{X}$ & & $\mathrm{X}$ & \\
\hline \multicolumn{6}{|l|}{ Participant safety } \\
\hline Adverse effects & & & $\mathrm{X}$ & $x$ & \\
\hline
\end{tabular}

\section{Patient safety}

Any adverse events (described as unfavourable or unintended signs, symptoms or diseases occurring after treatment) related to Tianjiu therapy will be observed and reported by patients and practitioners during each patient visit. In addition, all vital signs and adverse events will be measured and recorded at each visit.

\section{Sample size and statistical analysis}

To the best of the authors' knowledge, no randomised trials investigating the efficacy of Tianjiu therapy for IDH have been conducted to date. Sufficient data on estimates of the SD of the proposed outcome measures in this population are not available for use in the calculation of a sample size. This pilot trial is designed to collect such data to inform the efficacy and sample size of Tianjiu therapy for a larger definitive trial in the future.

To collect sufficient data to inform a future sample size calculation, we anticipate a $16 \mathrm{~mm} \mathrm{Hg}$ improvement due to Tianjiu therapy, and one of $0 \mathrm{~mm} \mathrm{Hg}$ due to sham Tianjiu therapy. Sample size calculations will be conducted using G-Power V.3.1, with an $\alpha$ value of 0.05 and power of $80 \% .^{30}$ The dropout rate during the study is estimated to be $10 \%$, so a minimum of 45 participants will be needed in each group.

Baseline variables will be compared with the $\chi^{2}$ test for dichotomous variables and the t test or Wilcoxon rank sum test for continuous variables. Primary and secondary outcome measures will be compared with the paired t test or Wilcoxon signed rank test as appropriate. Differences in the degree of fatigue after dialysis and recovery time from fatigue after dialysis between pretest and post-test will be analysed by Bowker's test. Differences will be considered statistically significant when the $p$ value is $<0.05$. Analyses will be performed using SPSS V.18.0 and Microsoft Excel.

\section{DISCUSSION}

This trial is expected to provide convincing evidence that Tianjiu therapy has an effect for treating IDH. Extant literature shows that moxibustion is effective for patients with $\mathrm{HD}$; however, actual clinical practice has aspects that are somewhat difficult to overcome, such as unpleasant odours, burning and blistering, skin lesions or other physical disturbances. ${ }^{31}$ With regard to this situation, Tianjiu therapy can prevent the above complications and induce greater increases in skin temperature and blood perfusion than single moxibustion. ${ }^{32}$ In addition, stimulating the acupoints on the affected meridians can produce specific effects on regulating the corresponding organs. ${ }^{33}$

Many new drugs and dialysis techniques to control IDH have been developed, with all being administered during the HD itself. However, they still cannot effectively combat the side effects of IDH because of coexisting factors such as heart disease, diabetes, old age, atherosclerosis and impaired sympathetic response. ${ }^{34}$ 
These factors should therefore also be considered in patients prone to IDH, regardless of UF volume. Thermal therapy through acupoints to restore the balance between Yin and Yang has been shown to improve vitality in patients with HD. ${ }^{35}$ There is also evidence that the use of Tianjiu on the Lung-Qi tonifying acupoints such as BL12 (Fengmen), BL13 (Feishu) and GV14 (Dazhui) can reduce the symptoms of allergic rhinitis and regulate the autonomous nervous system in patients with asthma. ${ }^{36} 37$ On the basis of our experience in clinical practice, Tianjiu therapy with pasting on acupoints to activate Yang-Qi could reduce the fatigue of patients with HD. Few clinical trials have been carried out to evaluate the efficacy of herb paste for HD. Thus, we designed a short-term Tianjiu therapy at CV4 and KI1 to determine the regulatory effect for patients with IDH. Our methods for recruitment, randomisation, allocation, dialysis intervention, outcome assessment and data collection methods have been described in detail. Other assessments including more suitable acupoint applications, long-term therapeutic courses and data on the efficacy of different TCM syndromes in patients with IDH will also be considered in a subsequent study.

To maximally exclude the placebo effect, rigorous methodological designs are needed. ${ }^{38}$ In previous studies, no examples have used valid placebo or sham methods of acupoint herb paste. However, sham-Tianjiu can be practical for blinding purposes because of the rarity of experience with Tianjiu among patients with HD. For pragmatic purposes, we plan to use brown clay patches as a sham device.

In conclusion, this pilot, single-blinded RCT will investigate the efficacy and safety of Tianjiu for IDH, assess the feasibility and relevance of a Tianjiu therapy study design, and provide a clinical foundation for future large-scale pluralistic clinical trials.

\section{Author affiliations \\ ${ }^{1}$ Graduate Institute of Integrated Medicine, College of Chinese Medicine, China Medical University, Taichung, Taiwan \\ ${ }^{2}$ Department of Chinese Medicine, Kaohsiung Chang Gung Memorial Hospital and Chang Gung University College of Medicine, Kaohsiung, Taiwan ${ }^{3}$ Division of Nephrology, Department of Internal Medicine, Kaohsiung Chang Gung Memorial Hospital and Chang Gung University College of Medicine, Kaohsiung, Taiwan \\ ${ }^{4}$ School of Chinese Medicine for Post Baccalaureate, I-Shou University, Kaohsiung, Taiwan \\ ${ }^{5}$ School of Chinese Medicine, College of Chinese Medicine, China Medical University, Taichung, Taiwan \\ ${ }^{6}$ Department of Psychology, College of Medical and Health Science, Asia University, Taichung, Taiwan}

Acknowledgements The authors would like to express our gratitude to the people in the outpatient dialysis units and Chinese Medicine Pharmacy of the Physical Building of Chang Gung Memorial Hospital for their full cooperation and material support. The authors are also grateful to Dr. Hung-Huan Ma for his professional assistance. This trial was financially supported by the Chang Gung Memorial Hospital with grant number CMRPG 8D0341. This work was also supported in part by China Medical University (CMU104-S-37), Taiwan Ministry of Science and Technology (MOST 104-2320-B-039-016-MY3), and the Taiwan Ministry of Health and Welfare Clinical Trial and Research Center of Excellence (MOHW105-TDU-B-212-133019).
Contributors $\mathrm{M}-\mathrm{YT}$ and $\mathrm{C}-\mathrm{HW}$ conceived this trial and participated in the design of this trial. M-YT, S-YC and Y-CH planned the data analysis and drafted the manuscript. Y-JS and $\mathrm{H}-\mathrm{YN}$ will coordinate the trial and contribute to the screening of patients. C-HW, Y-JS and H-YN are involved in the recruitment of participants from clinics. C-HW and $\mathrm{Y}-\mathrm{HC}$ are responsible for the design and supervision of the study and the revision of the manuscript. All authors have read and approved the final manuscript.

Competing interests None declared.

Patient consent Obtained.

Ethics approval The ethics review board of Chang Gung Memorial Hospital.

Provenance and peer review Not commissioned; externally peer reviewed.

Data sharing statement The results of this pilot study will be disseminated via peer-reviewed publications and conference presentations. All of the data are available.

Open Access This is an Open Access article distributed in accordance with the Creative Commons Attribution Non Commercial (CC BY-NC 4.0) license, which permits others to distribute, remix, adapt, build upon this work noncommercially, and license their derivative works on different terms, provided the original work is properly cited and the use is non-commercial. See: http:// creativecommons.org/licenses/by-nc/4.0/

\section{REFERENCES}

1. Sherman RA, Daugirdas JT, Ing TS. Complications during hemodialysis. In: Daugirdas JT, Blake PG, Ing TS, eds. Handbook of dialysis. 4th edn. Philadelphia: Lippincott Williams and Wilkins, 2011:170-91.

2. Laupacis A, Muirhead N, Keown P, et al. A disease-specific questionnaire for assessing quality of life in patients on hemodialysis. Nephron 1992;60:302-6.

3. K/DOQI Workgroup. K/DOQI clinical practice guidelines for cardiovascular disease in dialysis patients. Am J Kidney Dis 2005;45 (Supplement 3):1-153.

4. Schreiber MJ Jr. Setting the stage. Am J Kidney Dis 2001;38 (Supplement 4):1-10.

5. Hekmat R, Ahmadi M, Fatehi $\mathrm{H}$, et al. Correlation between asymptomatic intradialytic hypotension and regional left ventricular dysfunction in hemodialysis patients. Iran J Kidney Dis 2011;5:97-102.

6. Bogaard HJ, de Vries JP, de Vries PM. Assessment of refill and hypovolaemia by continuous surveillance of blood volume and extracellular fluid volume. Nephrol Dial Transplant 1994;9:1283-7.

7. Flythe JE, Kimmel SE, Brunelli SM. Rapid fluid removal during dialysis is associated with cardiovascular morbidity and mortality. Kidney Int 2011:79:250-7.

8. Daugirdas JT. Dialysis hypotension: a hemodynamic analysis. Kidney Int 1991;39:233-46.

9. Mc Causland FR, Prior LM, Heher E, et al. Preservation of blood pressure stability with hypertonic mannitol during hemodialysis initiation. Am J Nephrol 2012;36:168-74.

10. Rubinger D, Backenroth R, Sapoznikov D. Sympathetic nervous system function and dysfunction in chronic hemodialysis patients. Semin Dial 2013;26:333-43.

11. Raj DS, Vincent B, Simpson K, et al. Hemodynamic changes during hemodialysis: role of nitric oxide and endothelin. Kidney Int 2002;61:697-704.

12. Agarwal R. How can we prevent intradialytic hypotension? Curr Opin Nephrol Hypertens 2012;21:593-9.

13. Palmer BF, Henrich WL. Recent advances in the prevention and management of intradialytic hypotension. J Am Soc Nephrol 2008;19:8-11.

14. Tai DJ, Conley J, Ravani P, et al. Hemodialysis prescription education decreases intradialytic hypotension. J Nephrol 2013:26:315-22.

15. Zhou YL, Liu HL, Duan XF, et al. Impact of sodium and ultrafiltration profiling on haemodialysis-related hypotension. Nephrol Dial Transplant 2006;21:3231-7.

16. Duan L, Zhao G, Ji B, et al. Effect of crude-herb moxibustion on blood lipids in rats with dyslipidemia. J Tradit Chin Med Sci 2014;1:140-7.

17. Yue ZH, He XQ, Chang XR, et al. The effect of herb-partition moxibustion on Toll-like receptor 4 in rabbit aorta during atherosclerosis. J Acupunct Meridian Stud 2012;5:72-9. 
18. Kondo $\mathrm{T}$, Kawamoto M. Acupuncture and moxibustion for stress-related disorders. Biopsychosoc Med 2014;8:7.

19. Han JB, Oh SD, Lee KS, et al. The role of the sympathetic nervous system in moxibustion-induced immunomodulation in rats. $J$ Neuroimmunol 2003;140:159-62.

20. Lin JG, Chen YH. The mechanistic studies of acupuncture and moxibustion in Taiwan. Chin J Integr Med 2011;17:177-86.

21. Noguchi E, Ohsawa H, Takagi K. Neural mechanism of localized changes in skeletal muscle blood flow caused by moxibustion-like thermal stimulation of anesthetized rats. J Physiol Sci 2009;59:421-7.

22. Sun H, Qiu MY, Li BQ, et al. Effect of moxibustion on quality of life in hemodialysis patients. Zhongguo Zhen Jiu 2008;28:321-4.

23. Li N, Qiu MY, Hao JR, et al. Randomized controlled trail on moxibustion for maintenance hemodialysis patients in deficiency syndrome. Zhongguo Zhen Jiu 2011;31:15-18.

24. Varon J. The diagnosis and treatment of hypertensive crises. Postgrad Med 2009;121:5-13.

25. Daugirdas JT. Second generation logarithmic estimates of single-pool variable volume $\mathrm{Kt} / \mathrm{V}$ : an analysis of error. J Am Soc Nephrol 1993;4:1205-13.

26. Tian $\mathrm{CH}$. Tian's Clinical Experiences. Beijing: Sino-Culture Press, 2000:301-10.

27. Yang $X$, Xiong $X$, Yang G, et al. Effectiveness of stimulation of acupoint KI 1 by artemisia vulgaris (Moxa) for the treatment of essential hypertension: a systematic review of randomized controlled trials. Evid Based Complement Alternat Med 2014;2014:187484.

28. Chang XR, Hong J, Yi SX. Internal medicine conditions. In: Dragon $\mathrm{S}$, ed. 1st edn. Illustrated Chinese moxibustion techniques and methods. 2012:139-40.
29. Bian XM, Lv L, Lin WB, et al. Moxibustion therapy at CV4 prevents postoperative dysuria after procedure for prolapse and hemorrhoids. Evid Based Complement Alternat Med 2013;2013: 756095.

30. Johanson GA, Brooks GP. Initial scale development: sample size for pilot studies. Educ Psychol Meas 2010;70:394-400.

31. Kim SY, Chae Y, Lee SM, et al. The effectiveness of moxibustion: an overview during 10 years. Evid Based Complement Alternat Med 2011;2011:306515.

32. Noh SH, Lee BR, Yim YK. Single and triple moxibustion with large, indirect moxa induced differential effects on skin temperature and blood perfusion in healthy human participants: counterevidence to a previous report. Complement Ther Med 2014;22:311-19.

33. Zhao L, Chen J, Liu CZ, et al. A review of acupoint specificity research in China: status quo and prospects. Evid Based Complement Alternat Med 2012;2012:543943.

34. Tislér A, Akócsi K, Hárshegyi I, et al. Comparison of dialysis and clinical characteristics of patients with frequent and occasional hemodialysis-associated hypotension. Kidney Blood Press Res 2002;25:97-102.

35. Lin CH, Lee LS, Su LH, et al. Thermal therapy in dialysis patientsa randomized trial. Am J Chin Med 2011;39:839-51.

36. Hsu WH, Ho TJ, Huang CY, et al. Chinese medicine acupoint herbal patching for allergic rhinitis: a randomized controlled clinical trial. Am $J$ Chin Med 2010;38:661-73.

37. Li YM, Liu Q, Li XY. New percutaneous absorption herbal patch for asthma of paracmasis and its effect on the relative transcription factors of patients. Zhongguo Zhen Jiu 2012;32:459-63.

38. Park JE, Lee SS, Lee MS, et al. Adverse events of moxibustion: a systematic review. Complement Ther Med 2010;18:215-23. 